\title{
Correction to: Redefinition of the concept of fuzzy set based on vague partition from the perspective of axiomatization
}

\author{
Xiaodong $\operatorname{Pan}^{1} \cdot$ Yang $\mathrm{Xu}^{1}$
}

Published online: 7 February 2018

๑) Springer-Verlag GmbH Germany, part of Springer Nature 2018

\section{Correction to: Soft Comput}

https://doi.org/10.1007/s00500-017-2855-5

I recently found that several errors occur in the statement of Definition 5.2 in Section 5 in the paper "Redefinition of the concept of fuzzy set based on vague partition from the perspective of axiomatization".

As it had been pointed out at the end of Section 4 of this paper that "the set of vague attribute values is defined as a free algebra on the elementary set of vague attribute values", and fuzzy sets are mathematical formulation for vague attribute values, hence, the set of fuzzy sets in $U$ can be seen as freely generated by a vague partition of $U$.

Based on this consideration, Definition 5.2 of this paper can be corrected as follows:

Definition 5.2 Let $U=[a, b] \subset \mathbb{R}$ and $\widetilde{U}=\left\{\mu_{A_{1}}(x), \ldots\right.$, $\left.\mu_{A_{n}}(x)\right\}, n \in \mathbb{N}^{+}$, a vague partition of $U$. The set $\mathscr{F}(\widetilde{U})$ of fuzzy sets in $U$ with respect to $\widetilde{U}$ consists of the following elements:

(1) if there exists $i \in \bar{n}$ such that $\mu_{A}(x)=\mu_{A_{i}}(x)$ for all $x \in U$, then $A=\left\{\left(x, \mu_{A}(x)\right) \mid x \in U\right\} \in \mathscr{F}(\widetilde{U}) ;$

(2) if $\mu_{A}(x)=\bar{\mu}(x)=1$ for all $x \in U$, then $A=$ $\left\{\left(x, \mu_{A}(x)\right) \mid x \in U\right\} \in \mathscr{F}(\widetilde{U})$

(3) if $\mu_{A}(x)=\mu(x)=0$ for all $x \in U$, then $A=$ $\left\{\left(x, \mu_{A}(x)\right) \mid \bar{x} \in U\right\} \in \mathscr{F}(\widetilde{U}) ;$

The original article can be found online at https://doi.org/10.1007/ s00500-017-2855-5.

Xiaodong Pan

xdpan1@163.com

1 School of Mathematics, Southwest Jiaotong University, West Section, High-Tech Zone, Chengdu 611756, Sichuan,

People's Republic of China
(4) if $A=\left\{\left(x, \mu_{A}(x)\right) \mid x \in U\right\} \in \mathscr{F}(\widetilde{U})$ and $r \in \mathbb{Q}^{+}$, then $A^{r}=\left\{\left(x,\left(\mu_{A}(x)\right)^{r}\right) \mid x \in U\right\} \in \mathscr{F}(\widetilde{U}) ;$

(5) if $A=\left\{\left(x, \mu_{A}(x)\right) \mid x \in U\right\} \in \mathscr{F}(\widetilde{U})$, and $N$ is a strong negation on $[0,1]$, then $A^{N}=\left\{\left(x,\left(\mu_{A}(x)\right)^{N}\right) \mid x \in\right.$ $U\} \in \mathscr{F}(\widetilde{U})$;

(6) if $A=\left\{\left(\widetilde{U}, \mu_{A}(x)\right) \mid x \in U\right\}, B=\left\{\left(x, \mu_{B}(x)\right) \mid x \in\right.$ $U\} \in \mathscr{F}(\widetilde{U})$, and $\otimes$ is a triangular norm, then $A \cap_{\otimes} B=$ $\left\{\left(x, \mu_{A}(x) \otimes \mu_{B}(x)\right) \mid x \in U\right\} \in \mathscr{F}(\widetilde{U}) ;$

(7) if $A=\left\{\left(x, \mu_{A}(x)\right) \mid x \in U\right\}, B=\left\{\left(x, \mu_{B}(x)\right) \mid x \in\right.$ $U\} \in \mathscr{F}(\widetilde{U})$, and $\oplus$ is a triangular conorm, then $A \cup_{\oplus}$ $B=\left\{\left(x, \mu_{A}(x) \oplus \mu_{B}(x)\right) \mid x \in U\right\} \in \mathscr{F}(\widetilde{U}) ;$

(8) $\mathscr{F}(\tilde{U})$ not include other elements.

In fact, $\mathscr{F}(\widetilde{U})$ can be considered as a function space based on $\widetilde{U}$.

We apologize to the readers for any inconvenience these errors might have caused. 